\title{
Holomorphic quantization formula in singular reduction
}

\author{
Weiping Zhang*
}

\begin{abstract}
We show that the holomorphic Morse inequalities proved by Tian and the author $[\mathrm{TZ1}, 2]$ are in effect equalities by refining the analytic arguments in [TZ1, 2].
\end{abstract}

\section{$\S 0$. Introduction and the statement of main results}

Let $(M, \omega, J)$ be a compact Kähler manifold with the Kähler form $\omega$ and the complex structure $J$. Let $g^{T M}$ denote the corresponding Kähler metric. We make the assumption that there exists a Hermitian line bundle $L$ over $M$ admitting a Hermitian connection $\nabla^{L}$ such that $\frac{\sqrt{-1}}{2 \pi}\left(\nabla^{L}\right)^{2}=\omega$. Then $L$ admits a unique holomorphic structure so that $\nabla^{L}$ is the associated Hermitian holomorphic connection. We call $L$ the prequantum line bundle over $M$.

Next, suppose that $(M, \omega, J)$ admits a holomorphic Hamiltonian action of a compact connected Lie group $G$ with Lie algebra $\mathbf{g}$. Let $\mu: M \rightarrow \mathbf{g}^{*}$ be the corresponding moment map. Then a formula due to Kostant [K] (cf. [TZ1, (1.13)]) induces a natural $\mathbf{g}$ action on $L$. We make the assumption that this $\mathbf{g}$ action can be lifted to a holomorphic $G$ action on $L$. Then this $G$ action preserves $\nabla^{L}$. After an integration if necessary, we also assume that this $G$ action preserves the Hermitian metric on $L$. Then for any integer $p \geq 0$, the $p$-th Dolbeault cohomology $H^{0, p}(M, L)$ is a $G$-representation. We denote its $G$-invariant part by $H^{0, p}(M, L)^{G}$.

Now let $a \in \mathbf{g}^{*}$ be a regular value of $\mu$. Let $\mathcal{O}_{a} \subset \mathbf{g}^{*}$ be the coadjoint orbit of $a$. For simplicity, we assume that $G$ acts on $\mu^{-1}\left(\mathcal{O}_{a}\right)$ freely. Then the quotient space $M_{G, a}=\mu^{-1}\left(\mathcal{O}_{a}\right) / G$ is smooth. Also, $\omega$ descends canonically to a symplectic form $\omega_{G, a}$ on $M_{G, a}$ so that one gets the Marsden-Weinstein reduction $\left(M_{G, a}, \omega_{G, a}\right)$. Furthermore, the complex structure $J$ descends canonically to a complex structure $J_{G, a}$ on $T M_{G, a}$ so that $\left(M_{G, a}, \omega_{G, a}, J_{G, a}\right)$ is again Kähler. On the other hand, the pair $\left(L, \nabla^{L}\right)$ also descends canonically to a Hermitian holomorphic line bundle $L_{G, a}$ over $M$.

${ }^{*}$ Partially supported by the NNSF, SEC of China and the Qiu Shi Foundation. 
One of the purposes of this paper is to present an analytic proof of the following result.

Theorem 0.1. If $\mu^{-1}(0)$ is not empty, then there exists an open neighborhood $\mathbf{O}$ of $0 \in \mathbf{g}^{*}$ such that for any regular value $a \in \mathbf{O}$ of $\mu$ with $\mu^{-1}(a)$ nonempty, the following identity for Dolbeault cohomologies holds for any integer $p \geq 0$,

$$
\operatorname{dim} H^{0, p}(M, L)^{G}=\operatorname{dim} H^{0, p}\left(M_{G, a}, L_{G, a}\right) .
$$

In the case where $a=0 \in \mathbf{g}^{*}$ is a regular value of $\mu,(0.1)$ was proved by Guillemin-Sternberg [GS] for $p=0$, and recently for general $p$ by Teleman [T] in the algebraic situation, refining in this case the Morse type inequalities of Tian and Zhang [TZ1, Theorem 0.4]. In this case, (0.1) has also been extended by Wu [W] to cases where $M$ is non-compact, when the Lie group $G$ is Abelian.

While for the case where $0 \in \mathbf{g}^{*}$ is a singular value of $\mu$, Theorem 0.1 , of which a more general version has been proved by Teleman [T, Sect. 5] in the algebraic situation, refines the Morse type inequalities proved in [TZ2, Theorem 0.1].

In a recent preprint $[\mathrm{Br}]$, Braverman proposed an analytic proof of Teleman's result, in the regular reduction case, by extending the methods developed in [TZ1]. In particular, he pointed out that to get the equality (0.1) for the $a=0$ case, one needs to find a quasi-homomorphism between the Dolbeault complexes under considerations which verifies certain specific properties. Braverman actually constructed a quasi-homomorphism in his paper.

We combine the methods and results in [TZ1, 2] with Braverman's idea, that one needs to construct a suitable quasi-homomorphism, to prove Theorem 0.1. However, the quasi-homomorphism we will construct is different from the one in $[\mathrm{Br}]$, and ties closer to the methods in [TZ1]. We first prove an extension of (0.1), when $a=0$ is a regular value of $\mu$, to the case where the prequantum line bundle $L$ can be replaced by some more general vector bundles. We then prove Theorem 0.1 by using the methods in [TZ2].

The above arguments also lead to the following extension to the non-Abelian group action case of a result of Wu and Zhang [WZ, Corollary 4.8].

Theorem 0.2. If $a \in \mathbf{g}^{*}$ is a regular value of $\mu$ and $\mu^{-1}(a) \neq \emptyset$, then for any integer $p \geq 0$, the following identity holds,

$$
\operatorname{dim} H^{0, p}(M, \mathbf{C})=\operatorname{dim} H^{0, p}\left(M_{G, a}, \mathbf{C}_{G, a}\right) .
$$

This paper is organized as follows. In Section 1, we prove an extended holomorphic quantization formula, in the regular reduction case, to allow more general coefficients. In Section 2, we prove Theorems 0.1 and 0.2 .

\section{$\S 1$. An extended holomorphic quantization formula}


In this section, we extend the holomorphic quantization formula in $[\mathrm{T}]$ to allow more general coefficients. Our method is a combination of the method in $[\mathrm{TZ1}, 2]$ with an idea of Braverman $[\mathrm{Br}]$ that one needs to construct a quasihomomorphism between the Dolbeault complexes under considerations.

This section is organized as follows. In a), we state the main result of this section. In b), we recall the construction of an isometric embedding $J_{T}$ which has been essential to the arguments in [TZ1, 2]. In c), we recall some results in [TZ2] which include in particular Morse type inequalities valid for coefficients more general than the prequantum line bundle $L$. In d), we construct the above mentioned quasi-homomorphism. In e), we first prove an estimate verified by the quasi-isomorphism constructed in d), which can be thought of as an analogue of a result of Bismut and Lebeau [BL, (10.4)] in our situation. We then apply a trick of Braverman $[\mathrm{Br}]$ to complete the proof of the main result stated in a).

\section{a). An extended holomorphic quantization formula}

In this section, we assume that $0 \in \mathbf{g}^{*}$ is a regular value of the moment map $\mu$ with $\mu^{-1}(0) \neq \emptyset$ and, for simplicity, that $G$ acts on $\mu^{-1}(0)$ freely. To simplify the notation, we will denote $M_{G, 0}, \omega_{G, 0}$ etc. by $M_{G}, \omega_{G}$, etc..

Let $E$ be a $G$-equivariant Hermitian holomorphic vector bundle over $M$ with the Hermitian holomorphic connection denoted by $\nabla^{E}$. Then it induces canonically (cf. [TZ1]) a Hermitian holomorphic vector bundle $E_{G}$ over $M_{G}$.

Let $\mathbf{g}$ (and thus $\mathbf{g}^{*}$ also) be equipped with an $\mathrm{Ad} G$-invariant metric. Let $\mathcal{H}=|\mu|^{2}$ be the norm square of the moment map.

Let $h_{i}, 1 \leq i \leq \operatorname{dim} G$, be an orthonormal base of $\mathbf{g}^{*}$. Let $V_{i}, 1 \leq i \leq \operatorname{dim} G$, be the dual base of $h_{i}, 1 \leq i \leq \operatorname{dim} G$. Then we can write $\mu$ as

$$
\mu=\sum_{i=1}^{\operatorname{dim} G} \mu_{i} h_{i}
$$

with each $\mu_{i}$ a real function on $M$.

For any $V \in \mathbf{g}$, set円

$$
r_{V}^{E}=L_{V}^{E}-\nabla_{V}^{E}
$$

where $L_{V}^{E}$ denotes the infinitesimal action of $V$ on $E$.

Assumption 1.1. At any critical point $x \in M$ of $\mathcal{H}$, one has

$$
\sqrt{-1} \sum_{i=1}^{\operatorname{dim} G} \mu_{i}(x) r_{V_{i}}^{E}(x) \geq 0 .
$$

\footnotetext{
${ }^{1}$ We use the same notation $V$ to denote the vector field it generates on $M$.
} 
The main result of this section can be stated as follows, where we still use the superscript $G$ to denote the $G$-invariant part.

Theorem 1.2. If E verifies Assumption 1.1, then the following identity for Dolbeault cohomologies holds for any integer $p \geq 0$,

$$
\operatorname{dim} H^{0, p}(M, E)^{G}=\operatorname{dim} H^{0, p}\left(M_{G}, E_{G}\right) .
$$

Remark 1.3. Clearly, the prequantum line bundle $L$ verifies the Assumption 1.1. Thus (1.4) holds for $E=L$ and $E=\mathbf{C}$. That is, it contains the Teleman formula [T] and Theorem 0.2 in the case where $a=0$ is a regular value of $\mu$.

\section{b). An embedding from $\Omega^{0, *}\left(M_{G}, E_{G}\right)$ into $\Omega_{G}^{0, *}(M, E)$}

Let $\Omega^{0, *}(M, E)$ (resp. $\Omega^{0, *}\left(M_{G}, E_{G}\right)$ ) denote the set of smooth sections of $\wedge^{0, *}\left(T^{*} M\right) \otimes E\left(\right.$ resp. $\left.\quad \wedge^{0, *}\left(T^{*} M_{G}\right) \otimes E_{G}\right)$. We denote by $\Omega_{G}^{0, *}(M, E)$ the $G$ invariant part of $\Omega^{0, *}(M, E)$.

In this subsection, following [BL] and [TZ1], we construct explicitly, for any $T>0$, an isometric embedding $J_{T}: \Omega^{0, *}\left(M_{G}, E_{G}\right) \rightarrow \Omega_{G}^{0, *}(M, E)$.

As in [TZ1, Sect. 3b)], let $U$ be a sufficiently small $G$-invariant open neighborhood of $\mu^{-1}(0)$ such that $G$ acts on $U$ freely. Then $U / G$ is smooth and carries a canonically induced metric $g^{T(U / G)}$ (cf. [TZ1, (3.8)]).

Let $N_{G}$ be the normal bundle to $M_{G}$ in $U / G$. We identify $N_{G}$ as the orthogonal completement of $T M_{G}$ in $T(U / G)$, i.e., $\left.T(U / G)\right|_{M_{G}}=N_{G} \oplus T M_{G}$ and $\left.g^{T(U / G)}\right|_{M_{G}}=g^{N_{G}} \oplus g^{T M_{G}}$, where $g^{N_{G}}$ is the induced metric on $N_{G}$.

If $y \in M_{G}, Z \in N_{G, y}$, let $t \in \mathbf{R} \mapsto x_{t}=\exp _{y}^{U / G}(t Z) \in U / G$ be the geodesic in $U / G$ with $x_{0}=y,\left.\frac{d x_{t}}{d t}\right|_{t=0}=Z$. For $\varepsilon>0$, set $B_{\varepsilon}=\left\{Z \in N_{G} ;|Z|<\varepsilon\right\}$. Since $M_{G}$ is compact, there exists $\varepsilon_{0}>0$ such that for $0<\varepsilon<\varepsilon_{0}$, the map $(y, Z) \in N_{G} \mapsto \exp _{y}^{U / G}(Z) \in U / G$ is a diffeomorphism from $B_{\varepsilon}$ onto a tubular neighborhood $U_{\varepsilon}$ of $M_{G}$ in $U / G$. From now on, we identify $B_{\varepsilon}$ with $U_{\varepsilon}$ and use the notation $(y, Z)$ instead of $\exp _{y}^{U / G}(Z)$. In particular, we identify $y \in M_{G}$ with $(y, 0) \in N_{G}$.

Let $d v_{N_{G}}$ be the volume form of the fibers in $N_{G}$. Then $d v_{M_{G}}(y) d v_{N_{G}}(Z)$ is a natural volume form on the total space of $N_{G}$. Let $k(y, Z)$ be the smooth positive function on $B_{\varepsilon_{0}}$ defined by $d v_{U / G}(y, Z)=k(y, Z) d v_{M_{G}}(y) d v_{N_{G}}(Z)$. The function $k$ has a positive lower bound on $B_{\varepsilon_{0} / 2}$. Also, $k(y)=1$.

Now consider the fibration $G \rightarrow U \stackrel{\pi}{\rightarrow} U / G$. Let $h$ be the positive function on $U / G$ defined by $h(x)=\sqrt{\operatorname{vol}\left(\pi^{-1}(x)\right)}$ for any $x \in U / G$.

If $F$ is a $G$-invariant Hermitian vector bundle over $U$, then it induces canonically a Hermitian vector bundle $F_{U / G}$ over $U / G$ such that $\pi^{*} F_{U / G}=F$. We denote as in [TZ1, Sect. 3c)] by $\pi_{G}^{F}: \Gamma_{G}(F) \rightarrow \Gamma\left(F_{U / G}\right)$ the canonical isomorphism which maps a $G$-invariant section of $F$ to the corresponding section of $F_{U / G}$. We will 
usually omit the superscript $F$ from $\pi_{G}^{F}$ if there will be no confusion. If $\nabla^{F}$ is a $G$-invariant Hermitian connection on $F$, then it induces canonically a Hermitian connection $\nabla^{F_{U / G}}$ on $F_{U / G}$ (cf. [TZ1, (3.9)]).

Now as in [BL, Sect. 8g)], for $x=(y, Z) \in U_{\varepsilon_{0}}$, we identify $E_{U / G, x}$ (resp. $\left.\left(\wedge^{0, *}\left(T^{*} M\right)\right)_{U / G, x}\right)$ with $E_{U / G, y}\left(\operatorname{resp} . \quad\left(\wedge^{0, *}\left(T^{*} M\right)\right)_{U / G, y}\right)$ by parallel transport with respect to $\nabla^{E_{U / G}}$ (resp. $\nabla^{\left(\wedge^{0, *}\left(T^{*} M\right)\right)_{U / G}}$, with $\nabla^{\wedge^{0, *}\left(T^{*} M\right)}$ the Hermitian holomorphic connection on $\left.\wedge^{0, *}\left(T^{*} M\right)\right)$ along the geodesic $t \mapsto(y, t Z)$. The induced identification of $\left(\wedge^{0, *}\left(T^{*} M\right) \otimes E\right)_{U / G, x}$ with $\left(\wedge^{0, *}\left(T^{*} M\right) \otimes E\right)_{U / G, y}$ preserves the the Z-grading of $\left(\wedge^{0, *}\left(T^{*} M\right) \otimes E\right)_{U / G}$ and is $G$-equivariant.

Since $0 \in \mathbf{g}^{*}$ is a regular value of the moment map $\mu: M \rightarrow \mathbf{g}^{*}$, one verifies easily that $\mu^{-1}(0)$ is a nondegenerate submanifold, in the sense of Bott, of $\mathcal{H}=$ $|\mu|^{2}$. Let $d \mu:\left.T M\right|_{\mu^{-1}(0)} \rightarrow \mathbf{g}^{*}$ denote the restriction of the differential of $\mu$ on $\mu^{-1}(0)$. Clearly, for any $Z \in N_{G},\left.\pi^{*} Z \in T M\right|_{\mu^{-1}(0)}$ and $\left|d \mu\left(\pi^{*} Z\right)\right|$ depends only on $Z$.

Take $\varepsilon \in\left(0, \varepsilon_{0} / 2\right]$. Let $\rho: \mathbf{R} \rightarrow[0,1]$ be a smooth function such that $\rho(a)=1$ if $a \leq 1 / 2$, while $\rho(a)=0$ if $a \geq 1$. For $Z \in N_{G}$, set $\rho_{\varepsilon}(Z)=\rho(|Z| / \varepsilon)$.

For $T>0, y \in M_{G}$, set

$$
\alpha_{T}(y)=\int_{N_{G, y}} \exp \left(-T\left|d \mu\left(\pi^{*} Z\right)\right|^{2}\right) \rho_{\varepsilon}^{2}(Z) d v_{N_{G}}(Z) .
$$

Definition 1.4. For any $T>0$, let $J_{T}: \Omega^{0, *}\left(M_{G}, E_{G}\right) \rightarrow \Omega_{G}^{0, *}(M, E)$ be defined by

$$
J_{T}: \alpha \mapsto \pi^{*}\left(k^{-1 / 2} \alpha_{T}^{-1 / 2} h^{-1} \rho_{\varepsilon}(Z) \exp \left(-\frac{T\left|d \mu\left(\pi^{*} Z\right)\right|^{2}}{2}\right) \alpha\right) .
$$

One verifies easily that $J_{T}$ is well-defined and that it is an isometry from $\Omega^{0, *}\left(M_{G}, E_{G}\right)$ onto its image. In fact, although not explicitly written out, the map $J_{T}$ has played an essential role in [TZ1, 2], in particular, in getting the results in the following subsection.

\section{c). Deformations of Dolbeault complexes and Morse type inequali- ties}

In the rest of this section, we assume that $E$ verifies Assumption 1.1.

Let $\bar{\partial}^{E}$ (resp. $\bar{\partial}^{E_{G}}$ ) be the Dolbeault operator acting on $\Omega^{0, *}(M, E)$ (resp. $\left.\Omega^{0, *}\left(M_{G}, E_{G}\right)\right)$.

Following Tian and Zhang [TZ1, (1.21)], for any $T \in \mathbf{R}$, set

$$
\begin{gathered}
\bar{\partial}_{T}^{E}=e^{-T|\mu|^{2} / 2} \bar{\partial}^{E} e^{T|\mu|^{2} / 2}: \Omega^{0, *}(M, E) \rightarrow \Omega^{0, *}(M, E), \\
D_{T}^{E}=\sqrt{2}\left(\bar{\partial}_{T}^{E}+\left(\bar{\partial}_{T}^{E}\right)^{*}\right) .
\end{gathered}
$$


Set $\widetilde{h}=\left.h\right|_{M_{G}}$. Following [TZ1, (3.54)], set

$$
\begin{gathered}
\bar{\partial}_{Q}^{E_{G}}=\widetilde{h} \bar{\partial}^{E_{G}} \widetilde{h}^{-1}: \Omega^{0, *}\left(M_{G}, E_{G}\right) \rightarrow \Omega^{0, *}\left(M_{G}, E_{G}\right), \\
D_{Q}^{E_{G}}=\sqrt{2}\left(\bar{\partial}_{Q}^{E_{G}}+\left(\bar{\partial}_{Q}^{E_{G}}\right)^{*}\right) .
\end{gathered}
$$

By proceeding as in [TZ1, Sects. 2-4], one gets easily the following refinement of [TZ1, Theorems 3.13 (ii) and 4.2].

Proposition 1.5. There exist $c_{0}>0, T_{0}>0$ such that there are no nonzero eigenvalues of $D_{Q}^{E_{G}, 2}$ in $\left[0, c_{0}\right]$, and that for any $T \geq T_{0}$, the number of eigenvalues of $\left.D_{T}^{E, 2}\right|_{\Omega_{G}^{0, *}(M, E)}$ in $\left[0, c_{0}\right]$ equals to $\operatorname{dim}\left(\operatorname{ker} D_{Q}^{E_{G}}\right)$.

From Proposition 1.5 and the $\mathbf{Z}$-grading nature of the problem, one gets for any integer $p \geq 0$ the following Morse type inequalities proved in [TZ2, Theorem $2.1]$

$$
\operatorname{dim} H^{0, p}(M, E)^{G} \leq \operatorname{dim} H^{0, p}\left(M_{G}, E_{G}\right)
$$

d). A quasi-homomorphism $r:\left(\Omega_{G}^{0, *}(M, E), \bar{\partial}_{T}^{E}\right) \rightarrow\left(\Omega^{0, *}\left(M_{G}, E_{G}\right), \bar{\partial}_{Q}^{E_{G}}\right)$

In this subsection, we construct a quasi-homomorphism from $\left(\Omega_{G}^{0, *}(M, E), \bar{\partial}_{T}^{E}\right)$ to $\left(\Omega^{0, *}\left(M_{G}, E_{G}\right), \bar{\partial}_{Q}^{E_{G}}\right)$. Our quasi-homomorphism is different from the one constructed by Braverman in $[\mathrm{Br}]$.

Let $i: \mu^{-1}(0) \hookrightarrow M$ denote the canonical isometric embedding.

One verifies easily that the induced Hermitian holomorphic bundle $E_{G}$ from $E$ is given by $E_{G}=\left(i^{*} E\right)_{U / G}$ with its Hermitian holomorphic connection given

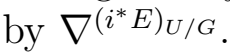

Let $N=\pi^{*} N_{G}$ be the normal bundle to $\mu^{-1}(0)$ in $M$. Then one verifies that $J N$ is the vertical tangent vector bundle of the fibration $G \rightarrow \mu^{-1}(0) \stackrel{\pi}{\rightarrow} M_{G}$ (cf. [TZ1, Sect. 3]). Thus one has the canonical orthogonal splittings

$$
\begin{aligned}
& \left.T M\right|_{\mu^{-1}(0)}=N \oplus J N \oplus \pi^{*}\left(T M_{G}\right), \\
& \left.g^{T M}\right|_{\mu^{-1}(0)}=g^{N} \oplus g^{J N} \oplus \pi^{*} g^{T M_{G}},
\end{aligned}
$$

with $J$ preserves $N_{J}=N \oplus J N$ and $W_{J}=\pi^{*}\left(T M_{G}\right)$. Thus one has as in [TZ1, (3.40)] the canonical identifications of Hermitian vector bundles

$$
\left.\wedge^{0, *}\left(T^{*} M\right)\right|_{\mu^{-1}(0)}=\wedge^{0, *}\left(N_{J}^{*}\right) \widehat{\otimes} \wedge^{0, *}\left(W_{J}^{*}\right) .
$$

Let $q$ be the canonical orthogonal projection

$$
q: \wedge^{0, *}\left(N_{J}^{*}\right) \widehat{\otimes} \wedge^{0, *}\left(W_{J}^{*}\right) \otimes i^{*} E \rightarrow \wedge^{0, *}\left(W_{J}^{*}\right) \otimes i^{*} E,
$$


which acts as identity on $\wedge^{0,0}\left(N_{J}^{*}\right) \otimes \wedge^{0, *}\left(W_{J}^{*}\right) \otimes i^{*} E \simeq \wedge^{0, *}\left(W_{J}^{*}\right) \otimes i^{*} E$ and maps each $\wedge^{0, i}\left(N_{J}^{*}\right) \otimes \wedge^{0, *}\left(W_{J}^{*}\right) \otimes i^{*} E, i \geq 1$, to zero.

Proposition 1.6. The following identity holds,

$$
\pi_{G} q i^{*} \bar{\partial}^{E}=\bar{\partial}^{E_{G}} \pi_{G} q i^{*}: \Omega_{G}^{0, *}(M, E) \rightarrow \Omega^{0, *}\left(M_{G}, E_{G}\right)
$$

Proof. For any $e \in T M$, write its complexification as $e=e^{1,0}+e^{1,0}$ with $e^{1,0} \in T^{(1,0)} M, e^{0,1} \in T^{(0,1)} M$. Let $c(e)$ be the Clifford action on $\wedge^{0, *}\left(T^{*} M\right) \otimes E$ defined by

$$
c(e)=\sqrt{2}\left({\overline{e^{1,0}}}^{*} \wedge-i_{e^{0,1}}\right),
$$

where ${\overline{e^{1,0}}}^{*} \in T^{(0,1) *} M$ is the metric dual of $e^{1,0}$. Then one verifies easily that

$$
c(J e)=-\sqrt{-2}\left({\overline{e^{1,0}}}^{*} \wedge+i_{e^{0,1}}\right) .
$$

Let $f_{1}, \cdots, f_{\operatorname{dim} M}$ be an orthonormal base of $T M$. Let $\nabla^{\wedge^{0, *}\left(T^{*} M\right) \otimes E}$ be the Hermitian holomorphic connection on $\wedge^{0, *}\left(T^{*} M\right) \otimes E$. Then one verifies easily that

$$
\bar{\partial}^{E}=\sum_{i=1}^{\operatorname{dim} M} \overline{f_{i}^{1,0}} \wedge \nabla_{f_{i}}^{\wedge^{0, *}\left(T^{*} M\right) \otimes E},
$$

by which one finds that if $e_{1}, \cdots, e_{\operatorname{dim} M_{G}}$ is an orthonormal base of $W_{J}$, then

$$
q i^{*} \bar{\partial}^{E}=\sum_{i=1}^{\operatorname{dim} M_{G}} \overline{e_{i}^{1,0}}{ }^{*} \wedge\left(i^{*} \nabla^{\wedge^{0, *}\left(T^{*} M\right) \otimes E}\left(e_{i}\right)\right) i^{*} .
$$

Now, let $P$ (resp. $P^{\perp}$ ) be the orthogonal projection from $\left.T M\right|_{\mu^{-1}(0)}$ to $N_{J}$ (resp. $W_{J}$ ) with respect to the orthogonal splitting (1.10). Set

$$
\nabla^{N_{J}}=P i^{*} \nabla^{T M} P, \quad \nabla^{W_{J}}=P^{\perp} i^{*} \nabla^{T M} P^{\perp},
$$

where $\nabla^{T M}$ is the Levi-Civita connection of $g^{T M}$, and

$$
A=i^{*} \nabla^{T M}-\nabla^{N_{J}}-\nabla^{W_{J}} .
$$

Then $A$ and $J$ commute with each other.

As in [TZ1, pp. 252], $\nabla^{N_{J}}, \nabla^{W_{J}}$ induce canonically the Hermitian connections $\nabla^{\wedge^{0, *}\left(N_{J}^{*}\right)}, \nabla^{\wedge^{0, *}\left(W_{J}^{*}\right)}$ on $\wedge^{0, *}\left(N_{J}^{*}\right), \wedge^{0, *}\left(W_{J}^{*}\right)$. Let ${ }^{0} \nabla^{\left.\left(\wedge^{0, *}\left(T^{*} M\right) \otimes E\right)\right|_{\mu^{-1}(0)}}$ denote the tensor product connection of $\nabla^{\wedge^{0, *}\left(N_{J}^{*}\right)}, \nabla^{\wedge^{0, *}\left(W_{J}^{*}\right)}$ and $i^{*} \nabla^{E}$. Then by [TZ1, (3.46)], one has that if $e_{\operatorname{dim} M_{G}+1}, \cdots, e_{\operatorname{dim} \mu^{-1}(0)}$ is an orthonormal base of $J N$, then for any $1 \leq i \leq \operatorname{dim} M_{G}$,

$$
i^{*} \nabla^{\wedge^{0, *}\left(T^{*} M\right) \otimes E}\left(e_{i}\right)={ }^{0} \nabla_{e_{i}}^{\left.\left(\wedge^{0, *}\left(T^{*} M\right) \otimes E\right)\right|_{\mu^{-1}(0)}}
$$




$$
+\frac{1}{2} \sum_{s=1}^{\operatorname{dim} M_{G}} \sum_{t=\operatorname{dim} M_{G}+1}^{\operatorname{dim} \mu^{-1}(0)}\left(\left\langle A\left(e_{i}\right) e_{s}, e_{t}\right\rangle c\left(e_{s}\right) c\left(e_{t}\right)+\left\langle A\left(e_{i}\right) e_{s}, J e_{t}\right\rangle c\left(e_{s}\right) c\left(J e_{t}\right)\right) .
$$

Now one verifies directly that

$$
\pi_{G} q \sum_{i=1}^{\operatorname{dim} M_{G}}{\overline{e_{i}^{1,0}}}^{*} \wedge\left({ }^{0} \nabla_{e_{i}}^{\left.\left(\wedge^{0, *}\left(T^{*} M\right) \otimes E\right)\right|_{\mu}-1(0)}\right) i^{*}=\bar{\partial}^{E_{G}} \pi_{G} q i^{*}
$$

On the other hand, by (1.14), (1.15) and the fact that $A$ and $J$ commute with each other, one deduces that

$$
\begin{aligned}
& \frac{1}{2} \sum_{s=1}^{\operatorname{dim} M_{G}} \sum_{t=\operatorname{dim} M_{G}+1}^{\operatorname{dim} \mu^{-1}(0)}\left(\left\langle A\left(e_{i}\right) e_{s}, e_{t}\right\rangle c\left(e_{s}\right) c\left(e_{t}\right)+\left\langle A\left(e_{i}\right) e_{s}, J e_{t}\right\rangle c\left(e_{s}\right) c\left(J e_{t}\right)\right) \\
= & \frac{1}{2} \sum_{s=1}^{\operatorname{dim} M_{G}} \sum_{t=\operatorname{dim} M_{G}+1}^{\operatorname{dim} \mu^{-1}(0)}\left(\left\langle A\left(e_{i}\right) e_{s}, e_{t}\right\rangle c\left(e_{s}\right) c\left(e_{t}\right)+\left\langle A\left(e_{i}\right) e_{s}, e_{t}\right\rangle c\left(J e_{s}\right) c\left(J e_{t}\right)\right) \\
& =-2 \sum_{s=1}^{\operatorname{dim} M_{G}} \sum_{t=\operatorname{dim} M_{G}+1}^{\operatorname{dim} \mu^{-1}(0)}\left\langle A\left(e_{i}\right) e_{s}, e_{t}\right\rangle\left(\overline{e_{s}^{1,0}}{ }^{*} \wedge i_{e_{t}^{0,1}}+i_{e_{s}^{0,1}}{\overline{e_{t}^{1,0}}}^{*} \wedge\right)
\end{aligned}
$$

Lemma 1.7. For any $\operatorname{dim} M_{G}+1 \leq t \leq \operatorname{dim} \mu^{-1}(0)$, one has,

$$
\sum_{i=1}^{\operatorname{dim}} \sum_{s=1}^{M_{G}}\left\langle A\left(e_{i}\right) e_{s}, e_{t}\right\rangle{\overline{e_{i}^{1,0}}}^{*} \wedge{\overline{e_{s}^{1,0}}}^{*}=0 .
$$

Proof. By the fibration structure of $G \rightarrow \mu^{-1}(0) \stackrel{\pi}{\rightarrow} M_{G}$, one knows that $\left[e_{i}, e_{t}\right]$ 's and $\left[J e_{i}, e_{t}\right]$ 's, with $1 \leq i \leq \operatorname{dim} M_{G}$, $\operatorname{dim} M_{G}+1 \leq t \leq \operatorname{dim} \mu^{-1}(0)$, are lying in $\Gamma(J N)$. Thus one has, since $A$ and $J$ commute with each other,

$$
\begin{gathered}
\left\langle A\left(e_{i}\right) e_{s}, e_{t}\right\rangle=\left\langle\nabla_{e_{i}} e_{s}, e_{t}\right\rangle=-\left\langle e_{s}, \nabla_{e_{i}} e_{t}\right\rangle=-\left\langle e_{s}, \nabla_{e_{t}} e_{i}\right\rangle \\
=-\left\langle J e_{s}, \nabla_{e_{t}} J e_{i}\right\rangle=\left\langle A\left(J e_{i}\right) J e_{s}, e_{t}\right\rangle .
\end{gathered}
$$

By (1.24), (1.14) and (1.15), one deduces that

$$
\begin{gathered}
\sum_{i=1}^{\operatorname{dim} M_{G}} \sum_{s=1}^{\operatorname{dim} M_{G}}\left\langle A\left(e_{i}\right) e_{s}, e_{t}\right\rangle{\overline{e_{i}^{1,0}}}^{*} \wedge{\overline{e_{s}^{1,0}}}^{*} \\
=\sum_{i=1}^{\operatorname{dim} M_{G}} \sum_{s=1}^{\operatorname{dim} M_{G}}\left\langle A\left(J e_{i}\right) J e_{s}, e_{t}\right\rangle \overline{\left(J e_{i}\right)^{1,0}}{ }^{*} \wedge \overline{\left(J e_{s}\right)^{1,0}} * \\
=-\sum_{i=1}^{\operatorname{dim} M_{G}} \sum_{s=1}^{\operatorname{dim} M_{G}}\left\langle A\left(e_{i}\right) e_{s}, e_{t}\right\rangle{\overline{e_{i}^{1,0}}}^{*} \wedge{\overline{e_{s}^{1,0}}}^{*},
\end{gathered}
$$

from which (1.23) follows. 
From (1.23) and (1.22), one finds that

$$
\begin{gathered}
q \sum_{i=1}^{\operatorname{dim} M_{G}} \sum_{s=1}^{\operatorname{dim} M_{G}} \sum_{t=\operatorname{dim} M_{G}+1}^{\operatorname{dim} \mu^{-1}(0)}\left(\left\langle A\left(e_{i}\right) e_{s}, e_{t}\right\rangle \overline{e_{i}^{1,0}}{ }^{*} \wedge c\left(e_{s}\right) c\left(e_{t}\right)\right. \\
\left.+\left\langle A\left(e_{i}\right) e_{s}, J e_{t}\right\rangle{\overline{e_{i}^{1,0}}}^{*} \wedge c\left(e_{s}\right) c\left(J e_{t}\right)\right) i^{*}=0
\end{gathered}
$$

From (1.17), (1.20), (1.21) and (1.26), one gets (1.13).

Definition 1.8. Let $r$ be the map defined by

$$
r=\widetilde{h} \pi_{G} q i^{*}: \Omega_{G}^{0, *}(M, E) \rightarrow \Omega^{0, *}\left(M_{G}, E_{G}\right) .
$$

Theorem 1.9. For any $T \in \mathbf{R}$, the map

$$
r:\left(\Omega_{G}^{0, *}(M, E), \bar{\partial}_{T}^{E}\right) \rightarrow\left(\Omega^{0, *}\left(M_{G}, E_{G}\right), \bar{\partial}_{Q}^{E_{G}}\right)
$$

is a quasi-homomorphism.

Proof. Let $X^{\mathcal{H}}$ be the Hamiltonian vector field associated with $\mathcal{H}=|\mu|^{2}$. By (1.7) one deduces that

$$
\bar{\partial}_{T}^{E}=\bar{\partial}^{E}+\frac{\sqrt{-1} T}{2}{\overline{\left(X^{\mathcal{H}}\right)^{1,0}}}^{*} \wedge .
$$

On the other hand, one verifies easily that

$$
\left.X^{\mathcal{H}}\right|_{\mu^{-1}(0)}=0
$$

From (1.8), (1.13), (1.27), (1.29) and (1.30), one gets

$$
r \bar{\partial}_{T}^{E}=\bar{\partial}_{Q}^{E_{G}} r: \Omega_{G}^{0, *}(M, E) \rightarrow \Omega^{0, *}\left(M_{G}, E_{G}\right),
$$

from which Theorem 1.9 follows.

\section{e). Proof of Theorem 1.2}

Recall that $c_{0}>0$ and $T_{0}>0$ have been fixed in Proposition 1.5. For any $T \geq T_{0}$, let $F_{G, T}^{\left[0, c_{0}\right]}$ be the finite dimensional subspace of $\Omega_{G}^{0, *}(M, E)$ generated by the eigenspaces of those eigenvalues of $\left.D_{T}^{E, 2}\right|_{\Omega_{G}^{0, *}(M, E)}$ which lie in $\left[0, c_{0}\right]$. Let $P_{T}$ denote the orthogonal projection from $\Omega_{G}^{0, *}(M, E)$ onto $F_{G, T}^{\left[0, c_{0}\right]}$.

Let $P_{Q}$ denote the orthogonal projection from $\Omega^{0, *}\left(M_{G}, E_{G}\right)$ to $\operatorname{ker}\left(D_{Q}^{E_{G}}\right)$.

We first prove the following analogue of [BL, (10.4)]. 
Theorem 1.10. There exist $\varepsilon>0, C>0, T_{1}>0$ such that for any $T \geq T_{1}$, any $\sigma \in \operatorname{ker}\left(D_{Q}^{E_{G}}\right)$,

$$
\left\|P_{Q} r P_{T} \sqrt{\alpha_{T}} J_{T} \sigma-\sigma\right\|_{0} \leq \frac{C}{\sqrt{T}}\|\sigma\|_{0} .
$$

Proof. We choose $T_{0}$ in Proposition 1.5 so that $c_{0}$ is not an eigenvalue of $\left.D_{T}^{E, 2}\right|_{\Omega_{G}^{0, *}(M, E)}, T \geq T_{0}$. Let $\delta$ be the circle of center 0 with radius $\sqrt{c_{0}}$ in $\mathbf{C}$. Then one has the following analogue of [BL, (10.6)],

$$
P_{T} J_{T} \sigma=\frac{1}{2 \pi \sqrt{-1}} \int_{\delta}\left(\lambda-\left.D_{T}^{E}\right|_{\Omega_{G}^{0, *}(M, E)}\right)^{-1} J_{T} \sigma d \lambda .
$$

One can also show that, by proceeding as in [BL, Sect. 9] and [TZ1, 2], for $T$ large enough and $\lambda \in \delta,\left\|\left(\lambda-\left.D_{T}^{E}\right|_{\Omega_{G}^{0 * *}(M, E)}\right)^{-1}\right\|_{\infty}$ is uniformly bounded.

The analogues of the above two facts in [BL, Proof of (10.4)] are all the ones in [BL, Proof of (10.4)] which were proved by using [BL, Theorem 9.25]. The point now is that here we need not an analogue of [BL, Theorem 9.25] to have these two properties. Proposition 1.5 is enough for our purpose.

On the other hand, the analogue of [BL, (10.29)], which now reads

$$
r \frac{1}{2 \pi \sqrt{-1}} \int_{\delta} \pi^{*}\left(h^{-1} \exp \left(-\frac{\left|d \mu\left(\pi^{*} Z\right)\right|^{2}}{2}\right) \frac{\sigma}{\lambda}\right) d \lambda=\sigma,
$$

clearly holds. One can then proceed as in [BL, Proof of (10.4)] to complete the proof of Theorem 1.10.

Proof of Theorem 1.2. We proceed as in [Br, Sect. 3].

First of all, from (1.32) one knows that

$$
P_{T} \sqrt{\alpha_{T}} J_{T}: \operatorname{ker}\left(D_{Q}^{E_{G}}\right) \rightarrow F_{G, T}^{\left[0, c_{0}\right]}
$$

is surjective when $T$ is very large. Combining with Proposition 1.5, we see that it is in fact an isomorphism.

Take $\alpha \in F_{G, T}^{\left[0, c_{0}\right]}$. Then $\bar{\partial}_{T}^{E} \alpha \in F_{G, T}^{\left[0, c_{0}\right]}$. By the above discussion, there exists $\beta \in \operatorname{ker}\left(D_{Q}^{E_{G}}\right)$ such that

$$
\bar{\partial}_{T}^{E} \alpha=P_{T} \sqrt{\alpha_{T}} J_{T} \beta
$$

From (1.36) and (1.31), one finds

$$
P_{Q} r P_{T} \sqrt{\alpha_{T}} J_{T} \beta=P_{Q} r \bar{\partial}_{T}^{E} \alpha=P_{Q} \bar{\partial}_{Q}^{E_{G}} r \alpha=0 .
$$

From (1.37) and (1.32), one finds

$$
\|\beta\|_{0} \leq \frac{C}{\sqrt{T}}\|\beta\|_{0},
$$


from which one sees that $\beta=0$ as $T$ is large enough.

Thus, when $T$ is large enough, one has that

$$
\left.\bar{\partial}_{T}^{E}\right|_{F_{G, T}^{\left[0, c_{0}\right]}}=0 .
$$

From (1.39) and Proposition 1.5, one sees that when $T$ is large enough,

$$
\operatorname{dim} \operatorname{ker}\left(\left.D_{T}^{E}\right|_{\Omega_{G}^{0, *}(M, E)}\right)=\operatorname{dim} F_{G, T}^{\left[0, c_{0}\right]}=\operatorname{dim} \operatorname{ker}\left(D_{Q}^{E_{G}}\right) .
$$

By (1.40) and (1.9), one completes the proof of Theorem 1.2.

\section{§2. Proof of Theorems 0.1 and 0.2}

This section is organized as follows. In a), we apply Theorem 1.2 and a trick in [TZ2] to prove Theorem 0.1. In b), we prove Theorem 0.2.

\section{a). Proof of Theorem 0.1}

We proceed as in [TZ2]. Take $a \in \mathbf{g}^{*}$. Since the coadjoint orbit $\mathcal{O}_{a}$ admits a canonical Kähler form $\omega_{a}$ and the holomorphical $\mathrm{Ad} G$ action on $\mathcal{O}_{a}$ is Hamiltonian with the moment map given by the canonical embedding $i_{a}: \mathcal{O}_{a} \hookrightarrow$ g* $^{*}$ (cf. [MS, Chap. 5]), the induced action of $G$ on the Kähler product $\left(M \times \mathcal{O}_{a}, \omega \times\left(-\omega_{a}\right)\right)$ is also Hamiltonian with the moment map $\mu_{a}: M \times \mathcal{O}_{a} \rightarrow \mathbf{g}^{*}$ given by

$$
\mu_{a}(x, b)=\mu(x)-b .
$$

Set $\mathcal{H}_{a}=\left|\mu_{a}\right|^{2}$. The following result has been proved in [TZ2].

Lemma 2.1. (Tian-Zhang [TZ2, Prop. 1.2]) There exists an open neighborhood $\mathbf{O}$ of $0 \in \mathbf{g}^{*}$ such that if $a \in \mathbf{O}$ and $(x, b) \in M \times \mathcal{O}_{a}$ is a critical point of $\mathcal{H}_{a}$, then the following inequality for the inner product on $\mathbf{g}^{*}$ holds,

$$
\langle\mu(x)-b, \mu(x)\rangle \geq 0 .
$$

Now let $a \in \mathbf{O}$ be a regular value of $\mu$ and, for simplicity, that $G$ acts on $\mu^{-1}(a)$ freely. Then $0 \in \mathbf{g}^{*}$ is a regular value of $\mu_{a}$. Furthermore, one has the standard identification of the symplectic quotients

$$
\mu_{a}^{-1}(0) / G \equiv \mu^{-1}\left(\mathcal{O}_{a}\right) / G=M_{G, a}
$$

carrying the canonically induced Kähler form $\omega_{G, a}$.

Recall that $L$ is the holomorphic pre-quantum line bundle over $M$. Let $\pi$ denote the projection from $M \times \mathcal{O}_{a}$ to its first factor $M$. Let $\mathcal{L}=\pi^{*} L$ be the 
pull-back holomorphic Hermitian line bundle over $M \times \mathcal{O}_{a}$, with the Hermitian holomorphic connection denoted by $\nabla^{\mathcal{L}}$. Then the $G$ action on $L$ lifts canonically to a holomorphic $G$-action on $\mathcal{L}$. In particular, for any $V \in \mathbf{g}$, its infinitesimal action on $\mathcal{L}$ is, via the Kostant formula $[\mathrm{K}]$ (cf. [TZ1, (1.13)]) for the g-action on $L$, given by

$$
L_{V}^{\mathcal{L}}=\nabla_{V}^{\mathcal{L}}-2 \pi \sqrt{-1}\langle\mu \pi, V\rangle,
$$

from which one has, in using the notation in (1.2), that

$$
r_{V_{i}}^{\mathcal{L}}=-2 \pi \sqrt{-1} \mu_{i}(x)
$$

at any point $(x, b) \in M \times \mathcal{O}_{a}$. By (2.1), (2.5) and Lemma 2.1, one verifies that at any critical point $(x, b) \in M \times \mathcal{O}_{a}$ of $\mathcal{H}_{a}$,

$$
\sum_{i=1}^{\operatorname{dim} G} \sqrt{-1} \mu_{a, i}(x, b) r_{V_{i}}^{\mathcal{L}}(x, b)=2 \pi\langle\mu(x)-b, \mu(x)\rangle \geq 0 .
$$

On the other hand, one verifies directly that the induced line bundle $\mathcal{L}_{G}$ over $\mu_{a}^{-1}(0) / G$ is exactly the line bundle $L_{G, a}$ over $M_{G, a}=\mu^{-1}\left(\mathcal{O}_{a}\right) / G$.

One can then apply Theorem 1.2 to $M \times \mathcal{O}_{a}, \mu_{a}$ and $\mathcal{L}$ to get

$$
\operatorname{dim} H^{0, p}\left(M \times \mathcal{O}_{a}, \mathcal{L}\right)^{G}=\operatorname{dim} H^{0, p}\left(M_{G, a}, L_{G, a}\right)
$$

for any integer $p \geq 0$.

Furthermore, by the definition of $\mathcal{L}$, one verifies directly that

$$
\begin{aligned}
\operatorname{dim} H^{0, p}\left(M \times \mathcal{O}_{a}, \mathcal{L}\right)^{G} & =\sum_{i+j=p} \operatorname{dim} H^{0, i}(M, L)^{G} \cdot \operatorname{dim} H^{0, j}\left(\mathcal{O}_{a}, \mathbf{C}\right)^{G} \\
& =\operatorname{dim} H^{0, p}(M, L)^{G}
\end{aligned}
$$

with the last equality follows from the easy facts that

$$
\begin{gathered}
\operatorname{dim} H^{0,0}\left(\mathcal{O}_{a}, \mathbf{C}\right)=\operatorname{dim} H^{0,0}\left(\mathcal{O}_{a}, \mathbf{C}\right)^{G}=1, \\
\operatorname{dim} H^{0, j}\left(\mathcal{O}_{a}, \mathbf{C}\right)=0 \text { for } j \geq 1
\end{gathered}
$$

(0.1) follows from (2.7) and (2.8).

\section{b). Proof of theorem 0.2}

The main observation is that, in using the notation in (1.2), one has

$$
r_{V}^{\mathbf{C}} \equiv 0
$$

for any $V \in \mathbf{g}$. Thus $\mathbf{C}$ verifies the conditions of Theorem 1.2. Furthermore, (2.10) plays roles for $\mathbf{C}$ similar to what Lemma 2.1 plays for $\mathcal{L}$ in a). And one 
sees that to proceed the arguments in a) for $\mathbf{C}$, one no longer needs to assume that $a$ is close to 0 in $\mathbf{g}^{*}$. That is, one can show that for any regular value $a \in \mathbf{g}^{*}$ of $\mu$,

$$
\operatorname{dim} H^{0, p}(M, \mathbf{C})^{G}=\operatorname{dim} H^{0, p}\left(M_{G, a}, \mathbf{C}_{G, a}\right) .
$$

On the other hand, since $G$ is connected, one verifies easily that

$$
\operatorname{dim} H^{0, p}(M, \mathbf{C})=\operatorname{dim} H^{0, p}(M, \mathbf{C})^{G} .
$$

By (2.11) and (2.12), the proof of Theorem 0.2 is completed.

Remark 2.2. Using the arguments in this paper, one can also show that the 'weighted' Morse type inequalities proved in [TZ3] are in effect equalities.

Acknowledgements. We are indebted to Youliang Tian in an obvious way for the many ideas he shared with us through the joint works [TZ1, 2].

\section{References}

[BL] J.-M. Bismut and G. Lebeau, Complex immersions and Quillen metrics. Publ. Math. IHES. 74 (1991), 1-297.

[Br] M. Braverman, Cohomology of the Mumford quotient. Preprint, math.SG/9809146.

[GS] V. Guillemin and S. Sternberg, Geometric quantization and multiplicities of group representations. Invent. Math. 67 (1982), 515-538.

[K] B. Kostant, Quantization and unitary representations. in Modern Analysis and Applications. Lecture Notes in Math. vol. 170, Springer-Verlag, (1970), pp. 87-207.

[MS] D. Mcduff and D. Salamon, Introduction to Symplectic Topology. Clarendon Press, Oxford, 1995.

[T] C. Teleman, The quantization conjecture revisited. Preprint, math.AG/9808029.

[TZ1] Y. Tian and W. Zhang, An analytic proof of the geometric quantization conjecture of Guillemin-Sternberg. Invent. Math. 132 (1998), 229-259.

[TZ2] Y. Tian and W. Zhang, Holomorphic Morse inequalities in singular reduction. Math. Res. Lett. 5 (1998), 345-352.

[TZ3] Y. Tian and W. Zhang, Symplectic reduction and a weighted multiplicity formula for twisted $\mathrm{Spin}^{c}$-Dirac operators. Asian J. Math. To appear.

[W] S. Wu, A note on higher cohomology groups of Kähler quotients. Preprint, math.SG/9809192.

[WZ] S. Wu and W. Zhang, Equivariant holomorphic Morse inequalities III: nonisolated fixed points. Geom. Funct. Anal. 8 (1998), 149-178.

Nankai Institute of Mathematics, Nankai University, Tianjin 300071, P. R. China

e-mail address: weiping@sun.nankai.edu.cn 